\title{
Theoretical premises of a "three in one" therapeutic approach to treat immunogenic and nonimmunogenic cancers: a narrative review
}

\author{
Anastasia S. Proskurina ${ }^{1}$, Vera S. Ruzanova ${ }^{1,2} \wedge$, Alexandr A. Ostanin ${ }^{3}$, Elena R. Chernykh ${ }^{3}$, \\ Sergey S. Bogachev ${ }^{1 \wedge}$
}

${ }^{1}$ Institute of Cytology and Genetics, Siberian Branch of the Russian Academy of Sciences, Novosibirsk, Russia; ${ }^{2}$ Novosibirsk State University, Novosibirsk, Russia; ${ }^{3}$ Research Institute of Fundamental and Clinical Immunology, Novosibirsk, Russia

Contributions: (I) Conception and design: AA Ostanin, ER Chernykh, SS Bogachev; (II) Administrative support: ER Chernykh, SS Bogachev; (III) Provision of study materials or patients: AS Proskurina, VS Ruzanova; (IV) Collection and assembly of data: AS Proskurina, VS Ruzanova; (V) Data analysis and interpretation: AS Proskurina, VS Ruzanova, AA Ostanin; (VI) Manuscript writing: All authors; (VII) Final approval of manuscript: All authors.

Correspondence to: Sergey S. Bogachev. 10 Lavrentiev Ave., Novosibirsk 630090, Russia. Email: labmolbiol@mail.ru.

Objective: We describe experimental and theoretical premises of a powerful cancer therapy based on the combination of three approaches. These include (I) in situ vaccination (intratumoral injections of CpG oligonucleotides and anti-OX40 antibody); (II) chronometric or metronomic low-dose cyclophosphamide (CMLD CP)-based chemotherapy; (III) cancer stem cell-eradicating therapy referred to as Karanahan (from the Sanskrit kärana ["source"] + han ["to kill”]).

Background: In murine models, the first two approaches are particularly potent in targeting immunogenic tumors for destruction. In situ vaccination activates a fully fledged anticancer immune response via an intricate network of ligand-receptor-cytokine interactions. CMLD CP-based chemotherapy primarily targets the suppressive tumor microenvironment and activates tumor-infiltrating effectors. In contrast, Karanahan technology, being aimed at replicative machinery of tumor cells (both stem-like and committed), does not depend on tumor immunogenicity. With this technology, mice engrafted with ascites and/or solid tumors can be successfully cured. There is a significant degree of mechanistic and therapeutic overlap between these three approaches. For instance, the similarities shared between in situ vaccination and Karanaban technology include the therapeutic procedure, the cell target [antigen-presenting cells (APC) and dendritic cells (DC)], and the use of DNA-based preparations (CpG and DNAmix). Features shared between CMLD CP-based chemotherapy and Karanaban technology are the timing and the dose of the cytostatic drug administration, which lead to tumor regression.

Methods: The following keywords were used to search PubMed for the latest research reporting successful eradication of transplantable cancers in animal models that relied on approaches distinct from those used in the Karanahan technology: eradication of malignancy, cure cancer, complete tumor regression, permanently eradicating advanced mouse tumor, metronomic chemotherapy, in situ vaccination, immunotherapy, and others.

^ ORCID: Anastasia S. Proskurina, 0000-0002-7650-4331; Vera S. Ruzanova, 0000-0002-6836-5618; Alexandr A. Ostanin, 0000-0001-6895-938X; Elena R. Chernykh, 0000-0003-2346-6279; Sergey S. Bogachev, 0000-0002-2019-9382. 
Conclusion: We hypothesize, therefore, that very potent anticancer activity can be achieved once these three therapeutic modalities are combined into a single approach. This multimodal approach is theoretically curative for any type of cancer that depends on the presence of tumor-inducing cancer stem cells, provided that the active therapeutic components are efficiently delivered into the tumor and the specific biological features of a given patient's tumor are properly addressed. We expect this multimodal approach to be primarily applicable to late-stage or terminal cancer patients who have exhausted all treatment options as well as patients with inoperable tumors.

Keywords: In situ vaccination; chronometric/metronome low-dose chemotherapy; Karanaban technology; immunity; cancer stem cells

Submitted May 28, 2021. Accepted for publication Oct 14, 2021.

doi: $10.21037 /$ tcr-21-919

View this article at: https://dx.doi.org/10.21037/tcr-21-919

\section{Introduction}

Current experimental and clinical approaches to treat patients with various cancer types and degrees of disease progression include three basic techniques that do not depend on a specific target or molecule but rather rely on induction of an integral anticancer response. These are in situ vaccination, chronometric or metronomic low-dose cyclophosphamide (CMLD CP)-based chemotherapy, and a universal approach dubbed Karanahan technology, which primarily targets tumor-initiating stem cells (TISCs). It is becoming increasingly clear that taking into account the temporal dynamics of molecular events in the entire organism, organs, tissues, tumor, and tumor cells is the key to successful therapy (1). Also, the paradigm of the patient's individual immune status as a starting point for downstream immunotherapeutic interventions has been put posited (2). These novel concepts form the core of the proposed technology, which is based on the chronometric delivery of therapeutic agents to the tumor depending on the specific features of the tumor and immune cells as well as on the immune status of the patient.

This analysis was motivated by our experimental studies where we used the Karanaban technology to successfully treat mice engrafted with incurable and aggressive cancers, in addition to Krebs-2 and Ehrlich carcinomas. We searched PubMed for research papers reporting completely distinct curative approaches to treating similarly aggressive cancer models. We aimed to compare the underlying mechanisms and combine the strongest elements of each of the approaches into an integrated anticancer platform.

Our analysis of the literature indicated that there were very few studies reporting survival of laboratory animals engrafted with solid or ascites forms of cancer beyond 90-150 days that could maintain their fertility and ability to produce healthy offspring. In the vast majority of cases, evidence of anticancer activity was established as inhibited or delayed tumor growth or longer time to death of cancerengrafted animals.

We primarily focused on papers in which incurable forms of cancer were successfully treated in laboratory animals (3-8), and those with a completely developed curative approach were of particular interest (9-15).

An analysis of the available literature revealed three conceptually distinct approaches that are detailed in this review. These approaches are (I) in situ vaccination, aimed to expand the population of tumor-specific cytotoxic $\mathrm{T}$ cells and to initiate a systemic immune response that would have an abscopal effect and affect "nonvaccinated" cancer foci $(3,10,16)$; (II) CMLD CP-based chemotherapy, aimed to inactivate the immunosuppressive activity of tumor-resident myeloid-derived suppressor cells (MDSCs) $(5-8,17,18)$ and to restore the activity of anticancer immune cells; (III) our Karanaban technology, which induces the massive apoptosis of cancer cells and reduces cancer grafts by eradicating TISCs. Our analysis indicates that the first 2 approaches are applicable in the context of immunogenic tumors, whereas Karanahan demonstrates sustained activity regardless of the "hot" or "cold" immune status of the tumor (19).

All three approaches share some features, such as intratumoral delivery of drugs and systemic injection of moderate doses of cyclophosphamide (CP), which opens an opportunity to combine the strong attributes of these approaches.

We wondered which elements of these three curative 
anticancer approaches were unique in terms of their therapeutic activity yet could be logically combined to produce a significantly enhanced synergistic effect of the novel integrative multimodal platform. Taken together, these elements should ensure complete lysis of tumor foci due to the cell-cycle dependent metronomic delivery of $\mathrm{CP}$, reduction of the immunosuppressive activity of tumorassociated stroma, eradication of TISCs, and formation of the systemic antitumor immunity.

To reveal the latest progress in the area and generate the narrative review, a literature search was conducted in PubMed using keywords (eradication of malignancy, metronomic chemotherapy, in situ vaccination, immunotherapy, and others).

We present the following article in accordance with the Narrative Review reporting checklist (available at https:// dx.doi.org/10.21037/tcr-21-919).

\section{In situ vaccination}

Relatively novel immunotherapeutic approaches known as in situ vaccination are based on the simultaneous activation of a broad range of immune cells within a small area of the tumor, which results in both a local antitumor immune response and abscopal effects (i.e., affecting both the injected tumor and distant noninjected metastatic lesions). This contrasts with the traditional approaches that are based on the systemic administration of anticancer drugs, which typically activates only a single arm of immunity.

Multiple technological platforms exploiting the concept of in situ vaccination are actively tested in the clinic. These include oncolytic viruses, modulation of activity of tumorinfiltrating myeloid cells and lymphocytes, ligand-receptor blockade [intratumor checkpoint, tumor necrosis factor (TNF) signaling], local irradiation, local radiofrequency, ultrasound and cryoablation, injections of transformed cells, cytokine activation, tissue electroporation, and combinations thereof $(16,20-26)$.

Local immunotherapy has several important advantages over systemic immunotherapy. The first one is a significantly lower dose of the locally delivered drug, as compared to the dose required for systemic delivery, which in turn translates into lower toxicity. The second is that several independent approaches can be safely combined without the risk of developing uncontrolled toxicities (such as those observed following a cytokine storm) $(16,20)$. The third is that high local concentrations of the drug can be achieved, which results in stronger immune responses.
Nonetheless, the ultimate goals of the technological platforms listed above include boosting the activity of antigen-presenting cells (APC), production of a broad range of antigens, and blocking the suppressive activity of the tumor-associated microenvironment $(3,16)$. Technically, the approach includes intratumoral delivery of factors that irreversibly activate tumor-associated immune cells, which govern cancer cells lysis. As a result, a broad range of antigens and a vast repertoire of primed and activated effector CD8+ $\mathrm{T}$ cells are formed.

Several notable examples of such therapeutic approaches have been reported in the literature. These have demonstrated a pronounced efficacy in transplanted and induced cancer models. For instance, dendritic cell (DC) activation can be readily achieved by CpG oligonucleotides or TNF- $\alpha$ (3,27-29). Monoclonal antibodies as well as specific ligands have also been exploited as powerful anticancer agents. Antibodies that block programmed cell death protein 1/programmed deathligand 1 (PD1/PD-L1; broadly referred to as checkpoint inhibitors) have been described to prevent PD-1-mediated inhibitory signaling in cytotoxic $\mathrm{T}$ cells, thereby maintaining their activity in the otherwise immunosuppressive tumor microenvironment $(28,30,31)$. The glycolipid molecules alphagalactosylceramide ( $\alpha \mathrm{GalCer}$ ) and glucoronosylceramide have also been used in experimental cancer treatments. These molecules are analogous to the endogenous glycolipid known as isoglobotrihexosylceramide, which is a self-antigen complexed with the CD1d of APC. This complex is known to interact with a receptor found on the surface of natural killer T cells (NKT cells). This engagement leads to NKT activation and interferon gamma (IFN- $\gamma$ ) production (32).

Currently, antibodies and ligands for the molecules belonging to the TNF superfamily, for instance, antiOX40 antibodies (OX40) (3,27-29,33-35), are being actively explored as antitumor modalities (36-38). Multiple research teams have attempted to exploit the interaction between OX40 and other TNF family receptors to suppress cancer progression. The approaches that showed some potency include antibodies, various protein fusions, and OX40 agonists, such as RNA aptamers (39-41) and small molecules (42). These molecules also activated $\mathrm{T} 4+$ lymphocytes, causing their proliferation and IFN- $\gamma$ secretion, which, in turn, boosts the adaptive immune response. Experimental evidence indicates that broad anticancer immunity depends on the endowment of these molecules with $\mathrm{Fc}$ fragment moiety-either as a natural part of the antibody or as a fusion with the therapeutic protein of interest. 
One of the most spectacular examples of how in situ vaccination can help eradicate tumors in mouse models has been published by Sagiv-Barfi and colleagues (3). In this work, the synergistic action of intratumorally administered CpG and agonistic anti-OX40 antibody cured multiple hard-to-treat immunogenic tumors, both spontaneous and established.

\section{CMLD CP-based chemotherapy}

Recent reports have highlighted the ability of CP to remodel the tumor microenvironment, which is known to severely suppress the activity of tumor-infiltrating immune cells. As it turns out, CP exerts a stimulating effect on such cells, and significant progress in understanding the mechanism(s) of CP activity in this context has been achieved for the low-dose metronomic regimen.

As a monotherapy, standard high-dose CP injection in mice results in long-term (6-20) reduction of the numbers of circulating immune cells in the peripheral blood, bone marrow, and spleen $(43,44)$. CP also stimulates a protumor activity of tumor stroma cells, thereby leading to tumor growth and progression (45). This weakened immune control of the tumor ultimately results in the formation of TISC-derived cellular clones resistant to CP $(6,43,44,46-52,53)$. On the other hand, low-dose metronomic delivery of CP has been shown to stimulate immune cells, particularly tumor-infiltrating cells such as natural killer cells (NK), NKT, and DCs. Importantly, such a treatment regimen switches the polarization of tumor-associated macrophages and may result in tumor regression $(17,54)$.

The antitumor activity of CMLD CP-based chemotherapy is largely involves the remodeling of MDSCs and the mature myeloid cell network. Basically, it represents a regulatory type of therapy that remodels the tumor stroma and prepares the tumor for efficient targeting by the factors of innate and adaptive immunity. This feature of $\mathrm{CP}$ is presently considered the core of its therapeutic anticancer activity. Chemotherapy based on CMLD CP makes it possible to directly kill cancer cells and repeatedly disrupt the suppressive tumor milieu (45). Notably, lowdose metronomic chemotherapy is known to work well only for CP but not for other cytostatic drugs.

Presently, three major avenues of CP-based chemotherapy are being actively explored. These include various regimens of chronometric or metronomic low-dose delivery, synergistic activity of $\mathrm{CP}$ and $\mathrm{CpG}$ oligonucleotides, and the combination of $\mathrm{CP}$ with various antibodies and cytokines.
Several studies have focused on testing the sequence, dose, and frequency of chronometric or metronomic low-dose chemotherapy based on the administration $\mathrm{CP}$ alone or in combination with $\mathrm{CpG}$. These empirical studies share one goal: to cure the laboratory animals of cancer. For instance, Manrique and colleagues comprehensively investigated various regimens and doses of chemotherapeutic drugs, types of CpGs, injection schedules, sites of injections, and duration of chemotherapy courses (18). The optimal scheme was based on intraperitoneal injection of $200 \mathrm{mkg} / \mathrm{kg}$ CP every 7 days for 4-6 courses, and CpG $(5 \mathrm{mg} / \mathrm{kg})$ was delivered on day 3 following $\mathrm{CP}$ injection, as this time was essential for the recovery of bone marrow after CP chemotherapy (which was then followed with a 4-day rest period). It was found that the CP dose of $100 \mathrm{mg} / \mathrm{kg}$ was ineffective; the injection site was a nonfactor; 5-fluorouracil, irinotecan, sunitinib, temozolomide, gemcitabine, docetaxel, paclitaxel, oxaliplatin, and doxorubicin-both as a monotherapy and in combination with a metronomic co-delivery with $\mathrm{CpG}$ were either ineffective, toxic, or poorly tolerated.

In other studies, complete curing of mice engrafted with immunogenic tumors was observed as a result of developing a similar drug delivery schedule $(5,17)$. CP was injected every 6 or 12 days at a dose of $140-200 \mathrm{mg} / \mathrm{kg}$ (up to 4 courses), and CpG (100 m/kg per single intratumoral injection) was administered simultaneously with CP. Interestingly, daily low-dose CP-based chemotherapy had little, if any, antitumor effect, while chronometric or metronomic injection of CP every 6 days resulted in the recruitment of innate immune cells to the tumor and stimulated their activity $(5,17)$.

Chemotherapy based on CMLD CP results in the activation of various cells of innate immunity, leading to broad and multipronged anticancer effects. Activation of tumor-infiltrating lymphocytes is associated with stronger expression of various markers, such as Nkp46, Nkg2d, Prf1, Gzmb (NK1.1), Cd207 and Cd74 (DCs), and Cd86 (F4/80 and Emr1, macrophages). Interleukin 15 (IL-15) and IL18 are among the upregulated genes responsible for the development, proliferation, and cytotoxicity of NK cells. In the tumor, stronger B220 expression associated with killer interferon-producing DCs can be observed following CP treatment. These cells kill neighboring tumor cells, and engulf and present tumor antigens, thereby bridging the innate immune cell killing and activation of adaptive $\mathrm{T}$ and $\mathrm{B}$ cell responses (55). CP induces secretion of CCL21 by the tumor stroma, which attracts peripheral immune cells 
such as macrophages, NKs, NKTs, and DCs, which in turn act together to destroy the tumor (45,56-61). Low-dose CP treatment leads to an increase in DC progenitors in bone marrow. These cells then mature into active DCs, which can activate adaptive immunity. Higher levels of Tlr7 and Csf1 contribute to the maturation of DCs (56). Finally, Icam2 and Icam1, expressed by endothelial and immune cells, are required for the transendothelial migration of immune cells to the tumor site $(5,17,61)$.

There are two major cell types present in the tumor stroma that display protumor features; these include regulatory T cells (Tregs) and MDSCs. Nontoxic low-dose chemotherapy with $\mathrm{CP}$ renders cancer cells immunogenic due to elimination of suppressive Tregs and reversion of MDSC functionality towards an antitumor phenotype.

CD25+ FOXP3 + T lymphocytes are Tregs. They secrete TGF-beta 1, IL-10, IFN- $\gamma$, and IL-35, while expressing CTLA-4. The aforementioned cytokine and CTLA4/ CD68 interaction may suppress the activity of effector $\mathrm{T}$ cells and DCs. Chemotherapy based on CMLD CP is highly effective at breaking the suppressive function of lymphocytes and may eliminate such cells from the tumor microenvironment. Moreover, one of the striking features of CMLD CP-based chemotherapy is that Tregs and other T cell subpopulations are differentially affected. This is likely due to the failure of repair machinery in Tregs to restore chromatin breaks following interstrand crosslink induction by $\mathrm{CP}(62,63)$. Consequently, $\mathrm{CP}$ treatment results in the loss of Treg inhibitory activity or complete eradication of Tregs from the tumor, whereas effector $\mathrm{T}$ cells are spared and may thereby mediate a potent immune response.

The largest population of immune cells forming the tumor stroma is composed of MDSCs, which comprise CD11+Gr-1+ myeloid cells displaying the intermediate differentiation phenotype. MDSCs constitute $30 \%$ of the total number of bone marrow cells and $4 \%$ of all mononuclear cells of the spleen. Murine MDSCs are represented by two major subpopulations. Anti-Gr-1 antibodies (R B6-8C5) bind the same epitope on two distinct molecules, Lyc6G and Lyc6C, which are found on MDSCs. Thereby, two MDSC fractions known as granulocyte/ polymorphonuclear MDSCs (CD11b+Lyc6G+Lyc6Clow, similar to neutrophils in terms of morphology) and monocyte MDSCs (CD11b+Lyc6G-Lyc6Chigh, similar to monocytes) can be found. The first fraction may constitute up to $80 \%$ of all MDSCs. Monocyte MDSCs are largely represented by tumor-associated macrophages and may constitute up to $50 \%$ of the weight of tumor-associated stroma. In growing solid tumors, monocyte MDSCs are known to quickly differentiate into M2 tumor-associated macrophages (64-66). Both cell populations of MDSCs actively suppress immune cells, mostly tumor-infiltrating $\mathrm{T}$ cells. Various mechanisms are involved and include interaction between TIM-3 and galectin-9, ADAM17 and CD62L, arginase 1, iNOS, TGF-beta 1, IL-10, COX2, VEGF, TGF beta 1, and indoleamine-2-3-dioxigenase (IDO) among other factors (67-69).

Chemotherapy based on CMLD CP switches the properties of MDSCs from protumor to antitumor status. This primarily occurs via repolarization of tumor-resident M2 macrophages towards a M1 phenotype, which is associated with active secretion of lysozymes and stronger antibody-dependent cellular cytotoxicity (ADCC)mediated phagocytic activity $(61,70,71)$. Antitumor properties of MDSCs can be significantly enhanced by combining CMLD CP-based chemotherapy with CpG oligonucleotides, antitumor vaccine, or cytokines (72-75).

Thus, CMLD CP-based chemotherapy-used as a monotherapy or in combination with $\mathrm{CpG}$, vaccines, or cytokines-has the following therapeutic features that can destroy the tumor.

* Being a cytostatic drug, CP directly kills cancer cells by inducing aberrant mitosis and apoptosis.

- Besides displaying direct cytotoxicity, CMLD CPbased chemotherapy (both as a monotherapy and as combinations with various immunomodulating agents) activates innate and adaptive immunity, which contributes to tumor eradication.

Major events occurring during this therapy are as follows:

(I) Recruitment of immune cells from the periphery into the tumor focus.

(II) Activation of professional properties of tumorinfiltrating cells of innate and adaptive immunity.

(III) Elimination of tumor-associated T-regs.

(IV) Conversion of MDSCs from the protumor to antitumor phenotype, with M2-to-M1 transition of macrophages being prominent in this process.

This multipronged attack against the tumor forms the foundation of the high efficacy of CMLD CP-based chemotherapy in cancer patients.

\section{Karanahan technology}

Studies performed in the laboratory of induced cell processes at the Institute of Cytology and Genetics, Siberian 
A

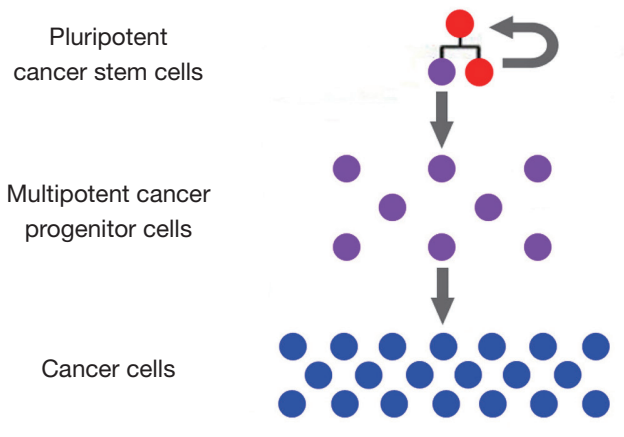

B
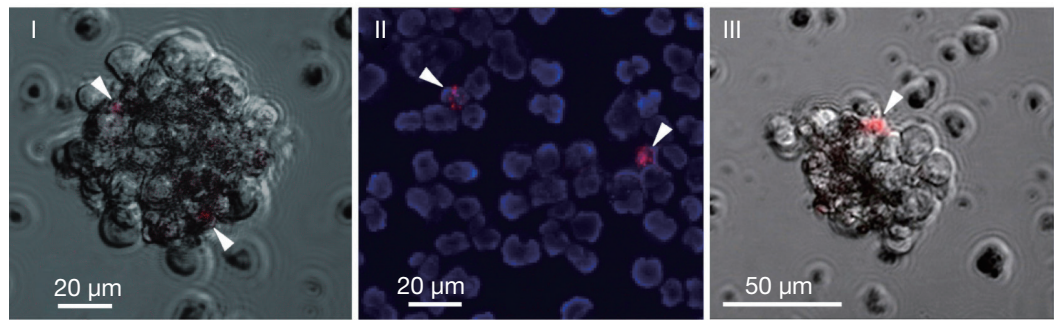

Figure 1 Cancer stem cells. (A) A model of cellular hierarchy within the tumor, with a self-perpetuating population of pluripotent cancer stem cells found on the top. (B) Various cancers encompass a subpopulation of DNA-internalizing cancer cells, referred to as TAMRA+ cancer stem cells (arrowheads): (I) sphere-forming cell line arising from human Epstein-Barr virus-induced lymphoma; (II) mouse Krebs-2 ascites; (III) a human lymphoma cell cluster. Cells $\left(10^{6}\right)$ were incubated with $0.1 \mathrm{mkg}$ TAMRA-labeled DNA fragment (human Alu repeat, about $500 \mathrm{bp}$ ) for 1 hour at room temperature in the dark.

Branch of the Russian Academy of Sciences (ICG SB RAS) have established a mechanism for the synergistic activity of $\mathrm{CP}$ and a composite double-stranded (dsDNA) preparation (DNAmix) against TISCs. These efforts have culminated in the development of a novel anticancer modality referred to as Karanahan technology ( कारणहन्, from the Sanskrit kärana ["source"] + han ["to kill"]). This approach primarily targets TISCs and induces massive apoptosis of committed tumor cells (6-8).

Karanaban technology is based on the three discoveries.

* Low-differentiated TISCs were found to share a universal molecular marker: they are capable of internalizing fragments of extracellular dsDNA. This feature can be conveniently exploited by using tetramethylrhodamine (TAMRA)-labeled DNA probes (Figure 1).

* Once internalized, these DNA fragments can potently interfere with the repair of interstrand DNA crosslinks produced by an earlier treatment with CP. This results in either direct TISC killing or in their loss of tumor-inducing properties. Without this supply of new tumor cells, the tumor is destroyed by the immune cells of an organism (3).
Within a given tumor, following CP treatment(s), synchronization of TISCs in their cell cycle can be achieved. Targeting them during a sensitive period of the cell cycle via the chronometric or metronomic delivery of cytotoxic agents may translate into the complete eradication of TISCs.

The process of repairing interstrand crosslinks after exposure to a crosslinking cytostatic, the main events occurring in the population of tumor cells following the application of Karanahan technology, and principles of $\mathrm{CP}$ and DNAmix administration within the Karanaban framework have been previously conceptualized in the figure published in a previous work (19).

This technology includes the following several steps:

(I) The percentage of low-differentiated TISCs within the tumor are measured (internalization of TAMRA+ DNA probe followed by FACS or microscopy analysis).

(II) The temporal profile of interstrand crosslink repair induced by the prior treatment with $\mathrm{CP}$ or mitomycin C (MMC) is clarified.

(III) The day when therapy-sensitive TAMRA+ TISCs synchronously enter the G1 phase of a cell cycle is 
identified-this occurs after 3 consecutive S/G2/M accumulation steps and is based on the information on the dynamics of the repair cycle.

(IV) DNAmix (6) is added to the therapy exactly at the timepoint that corresponds to the switching from the nucleotide excision repair (NER) to the homologous recombination (HR) phases so that a subfraction of the DNAmix molecules interferes with NER, and the rest of the molecules block HR. This prevents TISCs from counteracting the therapeutic "strike" and either kills them or renders them nontumorigenic. In parallel, addition of the DNAmix leads to a massive lysis of committed cancer cells.

This technology has been successfully tested on several experimental mouse and human cancers and in 3 pilot clinical cases $(6,8,76-79)$.

\section{Shared features of in situ vaccination, metronomic low-dose CP-based chemotherapy, and Karanahan technology, and the advantages of their combination into a single universal anticancer therapy}

The rationale behind this review is to open a discussion regarding whether the basic mechanisms underlying these 3 technologies can be safely combined into a novel approach that would be capable of inducing potent systemic anticancer immunity, reverting the immunosuppressive tumor microenvironment, inducing the apoptosis of differentiated cancer cells, and precipitating the eradication of TISCs.

In our opinion, this approach would perfectly complement the above three technologies and compensate for each of their weaknesses. Specifically, in situ vaccination would be improved by the introduction of an element that would render it efficient in the context of nonimmunogenic "cold" tumors. In turn, Karanahan technology incorporating the major features of the CMLD CP-based chemotherapy, namely the 6- to 7-day interval between the injections and the use of CP, would benefit from the induction of systemic antitumor immunity downstream of the receptor/ligandmediated APC activation.

\section{DNAmix and CP treatments are core elements of the Karanahan technology and CMLD CP-based chemotherapy}

At the core of Karanaban technology and CMLD CP- based chemotherapy is the use of DNAmix and CP. Their combination is rationally motivated and has been shown to be effective in "incurable" cancers, such as Krebs-2, U-87 xenografts, and B cell lymphomas in mice $(6,8,78,80,81)$.

It has been incontrovertibly shown that systemic anticancer immunity is boosted by such therapy $(6,8,77)$. Nonetheless, Karanaban technology is largely based on the eradication of TISCs and induction of massive apoptosis of committed cancer cells, rather than on anticancer immunity. This is supported by the following experimental data.

Only cancer cells comprising a TAMRA+ population of TISCs are capable of stable engraftment in mice. Cancer cells that are TISC-depleted (cell sorting or selective TISC killing by $\mathrm{CP}$ and DNAmix) fail to engraft in syngeneic or immunodeficient mice $(7,78)$. Notably, there is no contribution of the host immunity, as cytostatic drug treatment occurs ex vivo (another cross-linking cytostatic drug, MMC) prior to engraftment. Likewise, cell sorting has also been performed in cancer cell lines cultivated in vitro, so the observed failure to engraft can be attributed to the mouse immune system.

In our study, complete elimination of Krebs-2 tumors in mice could only be achieved via CP/DNAmix-based targeting of TISCs. This approach was also effective in a xenogeneic setting, with human U-87 glioblastoma cells being used as a model $(7,78)$. Our studies have firmly established that single or metronomic injections of $\mathrm{CP}$ and DNAmix are inefficient in treating such animals. This only becomes possible if the treatments are given to the animals at specific time points (depending on the dynamics of the DNA repair in each particular tumor) and when TISCs are completely eradicated once they become synchronized in a sensitive phase of the cell cycle. Even nonimmunogenic tumors can be eliminated this way. The same treatments performed without taking into account the repair dynamics invariably fail to fully cure the animals and lead to relapses $(6,8)$ despite activating the host immunity.

Indeed, Karanahan technology conveniently combines all the basic indicator parameters identified empirically for CMLD CP and CpG-based chemotherapy.

(I) $300 \mathrm{mg} / \mathrm{kg}$ of $\mathrm{CP}$ is given during the first 2 days, on days $5-9$ an additional (final) dose of $100 \mathrm{mg} / \mathrm{kg}$ is given vs. 140-200 mg/kg is given every 6-7 days (18);

(II) DNAmix is used as an activator of DCs vs. CpG activates the same APCs $(5,17)$.

(III) This means that use of Karanahan technology leads to the following key events, which are either unique or analogous to those observed 
after CMLD CP-based chemotherapy: TISCs are eradicated, differentiated cancer cells undergo apoptosis, suppressive activity of Tregs and MDSCs is abrogated, tumor-infiltrating lymphocytes are activated, and immune cells are recruited from the periphery.

\section{Principle of a combination of the three technologies}

Our analysis indicates that there are three major effects that may ultimately target the tumor for destruction.

(I) CpG combines with antibodies blocking checkpoint inhibitors or TNF family molecules. This treatment stimulates cytolytic activity of tumor-infiltrating immune cells and professional properties of DCs. It also helps destroy cancer cells and presents the broad range of cancer antigens to DCs "on site", which results in the induction of adaptive immunity.

(II) CMLD CP-based chemotherapy primarily targets the cellular components of a suppressive tumor microenvironment (Tregs, MDSCs). It also activates tumor-infiltrating immune cells and helps recruit periphery-derived, otherwise nonsuppressed immune cells into the tumor. When used in combination with $\mathrm{CpG}$ oligonucleotides, the effects are additively enhanced compared to those derived from a CP-only regimen.

(III) In Karanahan technology, the first $3 \mathrm{CP}$ injections over the course of 48-72 hours block proliferation of cancer cells and result in their massive apoptosis. When DNAmix is delivered during the specific timepoint of NER/HR phase, this additively enhances the apoptosis of committed cancer cells, drives TISCs towards an apoptotic pathway (as interstrand DNA crosslink repair is blocked), synchronizes TISCs in the G2/M(G1) phase of the cell cycle, activates the professional properties of DCs, and completely eradicates TISCs from the tumor. Injection of CP and DNAmix on days 5-9 provides the final "strike" to the surviving TISC population. In this setting, CP has a multifaceted activity: it alleviates tumor-infiltrating immune cell suppression, recruits peripheral immune cells, and activates all of the tumor-resident immune cells.

A combination of technologies makes sense when the weak aspects of one technology are compensated for by the strengths of the others and when the resulting approach is superior to any single technology used alone. There are 4 main aims of this combination: TISC eradication, apoptosis of differentiated cancer cells, alleviation of immune suppression, and activation of antitumor immunity.

Karanahan technology includes CMLD CP-based chemotherapy and has all the features of CMLD CP-based chemotherapy and $\mathrm{CpG}$ oligonucleotides. This indicates that Karanahan technology may entirely substitute CMLD CP-based chemotherapy, which targets T-regs and MDSCs for destruction, activates tumor-infiltrating immune cells including antigen-presenting DCs, and recruits peripheral immune cells into the tumor. The unique feature of Karanaban technology is that it allows complete TISC eradication, which leaves the tumor highly sensitive to the activated immune response.

Inclusion of immune checkpoint blockers or TNF family-specific antibodies (3) in DNAmix injections makes it possible to launch a fully fledged antitumor immune response mediated by ligand-receptor pathways. In this case, $\mathrm{CpG}$ is substituted for the DNAmix preparation, which essentially mimics all the effects of CpG.

There are several reasons why Karanahan technology may serve as an excellent unifying platform for two other anticancer approaches. The combination of Karanaban technology and in situ vaccination includes the use of DNAmix instead of CpG. An efficient dose of either agent may result in similar stimulation of the professional properties of DCs (82), the first component of in situ vaccination. In the context of Karanaban technology, DNAmix induces total apoptosis of committed cancer cells and launches the molecular events leading to the eradication of TISCs. The second component of in situ vaccination, namely the injection of OX40-specific antibodies, facilitates the stronger activation of the $\mathrm{T}$ cell response and may complement the therapeutic activity of the Karanaban technology with an adaptive immune response.

Once Karanahan technology and CMLD CP-based chemotherapy are combined, the common themes are (I) the similar therapeutic doses of CP and (II) the similar timing between the first 2 injections of CP in accordance with CMLD CP-based chemotherapy \{days 0-[6-7]\}, and the timing between tightly grouped, cell-cycle dependent triple injections of CP and the final "strike" injection that eradicates TISCs in accordance with Karanaban technology \{days 0-[4-9]\}.

Completion of the Karanaban technology therapeutic regimen leads to the induction of total apoptosis in 


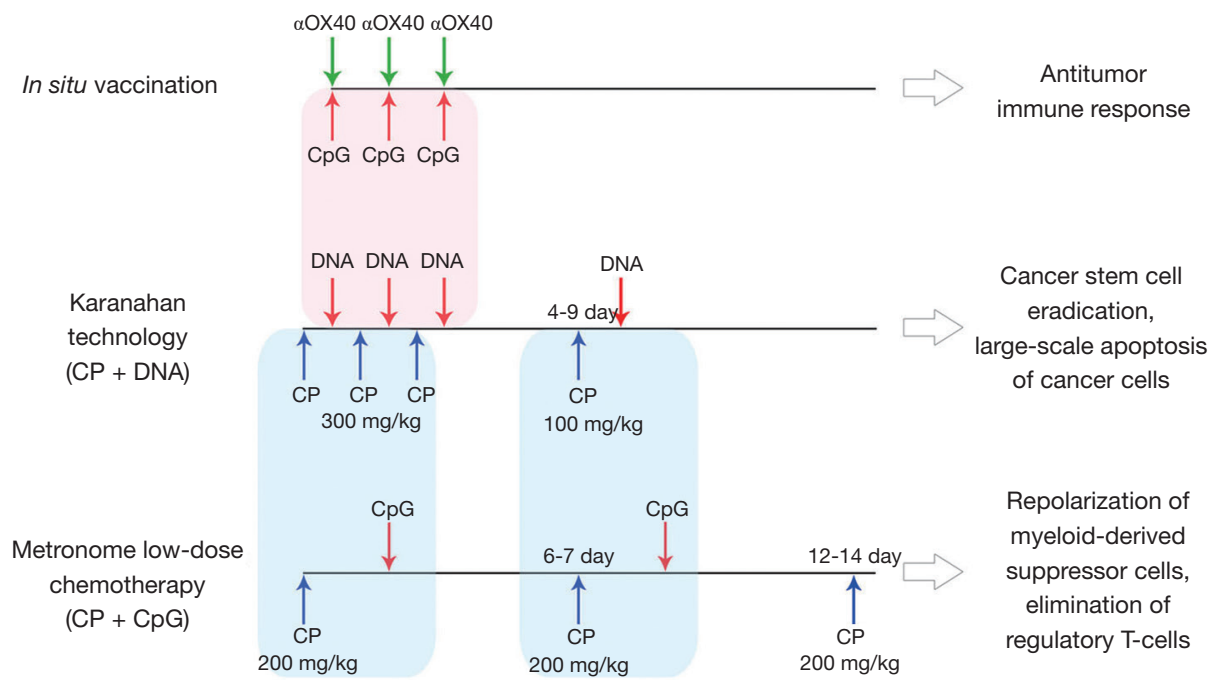

Figure 2 Side-by-side comparison of the three technologies. Shaded areas denote overlapping features of the approaches. $\alpha \mathrm{OX} 40$, antiOX40 antibodies; CpG, short synthetic single-stranded DNA molecules containing CpG motifs; CP, cyclophosphamide.

committed cancer cells and launches the events resulting in TISC elimination. The third injection of CP \{days 0-[4-9]$[12-14]\}$ (unless the tumor has been completely lysed by the preceding treatments according to Karanahan technology protocol) will serve to further strengthen the effect by eliminating the suppressive activity of the remaining tumorassociated stroma (Figure 2).

Once Karanaban technology is combined with just a single element of in situ vaccination platform and a single element of CMLD CP-based chemotherapy, 3 independent pathways to target the tumor are simultaneously induced. The cumulative therapeutic effect of such a combined approach leaves little likelihood that the primary tumor and the distant metastases will survive (Figure 3).

\section{Anticipated obstacles in the clinical implementation of the novel "three in one" anticancer platform}

Combining the three technologies may indeed cause problems with adapting the resulting platform to real-world clinical practice. This platform has a target population of stage 4 cancer patients for two reasons. First, this would be consistent with the current health care regulations in most countries. Second, our own studies of earlystage cancer samples indicate that such tumors display a discrete proliferative activity (83), which may complicate the accurate assessment of the cell cycle or repair duration as an input. Several technical issues exist as well. One is that some tumors are not accessible for intratumor injections due to their size and localization. In such cases, guided drug delivery may be considered. Second, different metastases may be heterogeneous in terms of their cell cycle parameters, which are key for the efficacy of Karanaban. This would necessitate the analysis of multiple biopsy specimens and a highly coordinated injection schedule. Third, delivery of the CP metabolite, phosphoramide mustard, is not uniform across different organs and tissues. Our limited clinical trial experience (data not shown) indicates that $\mathrm{CP}$ injections spare bone metastases. This is typically interpreted as the failure of phosphoramide mustard to efficiently reach this tissue, which is consistent with its poor blood supply. Karanahan monotherapy results in only partial response of liver-localized metastases, which may be attributable to insufficient concentration of the $\mathrm{CP}$ metabolite once it is produced in the liver and returns back from the circulation throughout the body. Ways to address this issue would be either to increase the $\mathrm{CP}$ dose or to provide ultrasound-guided delivery inside or adjacent to the tumor of directly alkylating drugs (nitrogen mustard, MMC). Injection of antibodies would facilitate the activation of $\mathrm{T}$ cell immunity, helping to eradicate a difficult-to-target cancer.

Activation of immunocompetent cells such as neutrophils and NK cells is of critical importance. Accurate timing of $\mathrm{CP}$ and DNAmix injection results in massive apoptosis of 


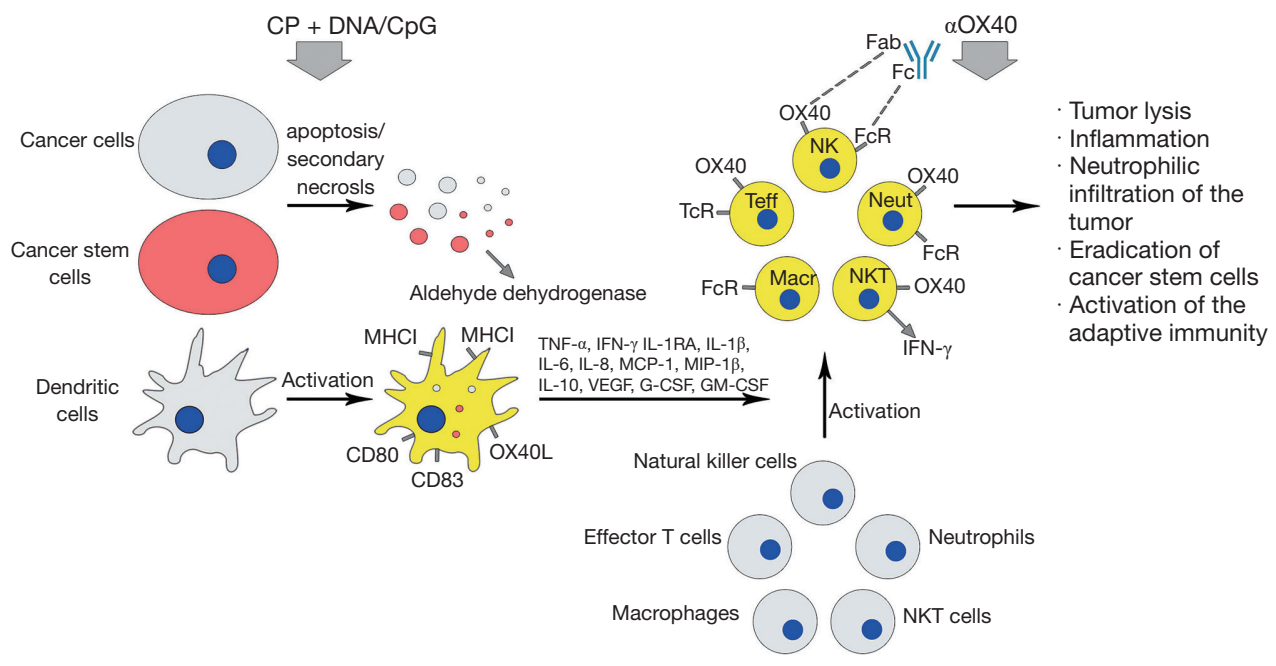

Figure 3 The progression of events induced by the integrated "three-in-one" technology. Successive intratumoral CP and DNAmix administration, strictly tied to the DNA repair timing, initiates the lysis of tumor cells that results in the formation of cellular debris consisting of apoptotic and necrotic components, elimination of cancer stem cells, disruption of the suppressive properties of tumorassociated stromal cells, development of local inflammation, and activation of the antigen-presenting properties of dendritic cells, which begin to secrete a wide range of cytokines (TNF- $\alpha$, IFN- $\gamma$, IL-1RA, IL-1 $\beta$, IL-6, IL-8, MCP-1, MIP-1 $\beta$, IL-10, VEGF, G-CSF, and GMCSF). These cytokines and direct cell-cell interactions activate tumor-infiltrating immune cells: effector T cells (Teff), NK, Neut, NKT, and Macr, which begin to express OX40 and FcR receptors on their surface. Anti-OX40 antibodies initiate a cascade of cellular and humoral reactions causing the development of adaptive immunity. CP, cyclophosphamide; NK, natural killer cells; Neut, neutrophils; NKT, natural killer T cells; Macr, OX40, macrophages; anti-OX40 antibodies.

cancer cells and subsequent secondary necrosis and tumor lysis, essentially forming a large inflammatory region that attracts neutrophils from the periphery. Chronometric delivery of CP also moderately potentiates NK cells (84) and antigen-presenting DCs. This results in the secretion of proinflammatory cytokines and growth factors, such as IL-1, TNF- $\alpha$, IFN- $\gamma$, and G-CSF $(85,86)$. DC activation in turn stimulates NKT cells and provides additional production of IFN- $\gamma(29,87)$. To summarize, all these events may culminate in a cytokine storm and systemic inflammatory reaction $(6,84,88)$. IFN- $\gamma$ and G-CSF/ GM-CSF stimulate surface expression of Fc receptors on neutrophils and NK cells. Consequently, delivery of OX40specific antibodies will not only boost $\mathrm{T}$ cell response but will also actively involve neutrophils and NK cells via $\mathrm{Fc} /$ FcR-mediated ADCC (33,89-92). Further upsurge of inflammation should follow $(33,93)$ and may manifest as multiple necrotic foci due to uncontrolled and nonspecific cytotoxic activity of neutrophils and NK cells, small vessel thrombosis, systemic inflammation, and multiple organ failure leading to death. Additional studies are required to comprehensively assess the likelihood of such a scenario. To overcome the above issues, transparent and easy-to-follow guidelines are needed for performing efficient resuscitation procedures and preventing such adverse reactions. This set of interventions may include the use of thrombolytic drugs and blood dialysis, as well as the administration of drugs that block renal, hepatic, and pulmonary insufficiency observed under severe sepsis conditions.

\section{Conclusions}

We hypothesize that very potent anticancer activity can be achieved once these three therapeutic modalities are combined into a single approach.

One factor that is key to the combination of the three technologies is timing. The mechanisms underlying any of these technologies should not interfere with each other. Specifically, one must estimate the time required to activate DCs using DNAmix, the time to activate innate and adaptive immunity by $\mathrm{OX} 40$-specific antibodies, the time needed for TISC eradication, and finally, the time required for total cancer cell apoptosis.

In principle, this "three-in-one" platform is envisaged 
to efficiently destroy any tumor that is dependent on the pool of TISCs. Whether or not it will work depends on how the technical issues and biological features of the tumor in a given patient are addressed. We strongly believe that this platform will be broadly used in terminal-stage cancer patients who are given maintenance support and in those patients with inoperable tumors. Tumor eradication or reduction of tumor burden open an opportunity for proceeding to the next line of therapy or therapy consolidation.

\section{Acknowledgments}

The authors are sincerely grateful to Andrey A. Gorchakov for the brilliant translation of the manuscript.

Funding: This work was supported by the Russian Ministry of Science and Higher Education via the Institute of Cytology and Genetics (State Budget Project No. 02592021-0013), the Russian Foundation for Basic Research (Project No. 18-29-09045), and "Karanahan" LLC.

\section{Footnote}

Reporting Checklist: The authors have completed the Narrative Review reporting checklist. Available at https:// dx.doi.org/10.21037/tcr-21-919

Conflicts of Interest: All authors have completed the ICMJE uniform disclosure form (available at https://dx.doi. org/10.21037/tcr-21-919). The authors have no conflicts of interest to declare.

Ethical Statement: The authors are accountable for all aspects of the work in ensuring that questions related to the accuracy or integrity of any part of the work are appropriately investigated and resolved.

Open Access Statement: This is an Open Access article distributed in accordance with the Creative Commons Attribution-NonCommercial-NoDerivs 4.0 International License (CC BY-NC-ND 4.0), which permits the noncommercial replication and distribution of the article with the strict proviso that no changes or edits are made and the original work is properly cited (including links to both the formal publication through the relevant DOI and the license). See: https://creativecommons.org/licenses/by-nc-nd/4.0/.

\section{References}

1. Adam D. Core Concept: Emerging science of chronotherapy offers big opportunities to optimize drug delivery. Proc Natl Acad Sci U S A 2019;116:21957-9.

2. Chen DS, Mellman I. Elements of cancer immunity and the cancer-immune set point. Nature 2017;541:321-30.

3. Sagiv-Barfi I, Czerwinski DK, Levy S, et al. Eradication of spontaneous malignancy by local immunotherapy. Sci Transl Med 2018;10:eaan4488.

4. Wu J, Waxman DJ. Immunogenic chemotherapy: Dose and schedule dependence and combination with immunotherapy. Cancer Lett 2018;419:210-21.

5. Jordan M, Waxman DJ. CpG-1826 immunotherapy potentiates chemotherapeutic and anti-tumor immune responses to metronomic cyclophosphamide in a preclinical glioma model. Cancer Lett 2016;373:88-96.

6. Potter EA, Dolgova EV, Proskurina AS, et al. A strategy to eradicate well-developed Krebs-2 ascites in mice. Oncotarget 2016;7:11580-94.

7. Dolgova EV, Alyamkina EA, Efremov YR, et al. Identification of cancer stem cells and a strategy for their elimination. Cancer Biol Ther 2014;15:1378-94.

8. Potter EA, Proskurina AS, Ritter GS, et al. Efficacy of a new cancer treatment strategy based on eradication of tumor-initiating stem cells in a mouse model of Krebs-2 solid adenocarcinoma. Oncotarget 2018;9:28486-99.

9. Lurje I, Werner W, Mohr R, et al. In Situ Vaccination as a Strategy to Modulate the Immune Microenvironment of Hepatocellular Carcinoma. Front Immunol 2021;12:650486.

10. Pierce RH, Campbell JS, Pai SI, et al. In-situ tumor vaccination: Bringing the fight to the tumor. Hum Vaccin Immunother 2015;11:1901-9.

11. Law AMK, Valdes-Mora F, Gallego-Ortega D. MyeloidDerived Suppressor Cells as a Therapeutic Target for Cancer. Cells 2020;9:561.

12. Varayathu H, Sarathy V, Thomas BE, et al. Combination Strategies to Augment Immune Check Point Inhibitors Efficacy - Implications for Translational Research. Front Oncol 2021;11:559161.

13. Simsek C, Esin E, Yalcin S. Metronomic Chemotherapy: A Systematic Review of the Literature and Clinical Experience. J Oncol 2019;2019:5483791.

14. Cazzaniga ME, Cordani N, Capici S, et al. Metronomic Chemotherapy. Cancers (Basel) 2021;13:2236. 
15. Chen J, Qiu M, Ye Z, et al. In situ cancer vaccination using lipidoid nanoparticles. Sci Adv 2021;7:eabf1244.

16. Marabelle A, Tselikas L, de Baere T, et al. Intratumoral immunotherapy: using the tumor as the remedy. Ann Oncol 2017;28:xii33-43.

17. Wu J, Waxman DJ. Metronomic cyclophosphamide schedule-dependence of innate immune cell recruitment and tumor regression in an implanted glioma model. Cancer Lett 2014;353:272-80.

18. Manrique SZ, Dominguez AL, Mirza N, et al. Definitive activation of endogenous antitumor immunity by repetitive cycles of cyclophosphamide with interspersed Toll-like receptor agonists. Oncotarget 2016;7:42919-42.

19. Ruzanova VS, Proskurina AS, Ritter GS, et al. Experimental Comparison of the In Vivo Efficacy of Two Novel Anticancer Therapies. Anticancer Res 2021;41:3371-87.

20. Bilusic M, Gulley JL. Editorial: Local Immunotherapy: A Way to Convert Tumors From "Cold" to "Hot". J Natl Cancer Inst 2017.

21. Aznar MA, Tinari N, Rullán AJ, et al. Intratumoral Delivery of Immunotherapy-Act Locally, Think Globally. J Immunol 2017;198:31-9.

22. Sersa G, Teissie J, Cemazar M, et al. Electrochemotherapy of tumors as in situ vaccination boosted by immunogene electrotransfer. Cancer Immunol Immunother 2015;64:1315-27.

23. Hammerich L, Binder A, Brody JD. In situ vaccination: Cancer immunotherapy both personalized and off-theshelf. Mol Oncol 2015;9:1966-81.

24. Hammerich L, Bhardwaj N, Kohrt HE, et al. In situ vaccination for the treatment of cancer. Immunotherapy 2016;8:315-30.

25. Murthy V, Minehart J, Sterman DH. Local Immunotherapy of Cancer: Innovative Approaches to Harnessing Tumor-Specific Immune Responses. J Natl Cancer Inst 2017.

26. Takahashi Y, Matsutani N, Nakayama T, et al. Immunological effect of local ablation combined with immunotherapy on solid malignancies. Chin J Cancer 2017;36:49.

27. Murphy KA, Erickson JR, Johnson CS, et al. CD8+ T cell-independent tumor regression induced by Fc-OX40L and therapeutic vaccination in a mouse model of glioma. $\mathrm{J}$ Immunol 2014;192:224-33.

28. Jensen SM, Maston LD, Gough MJ, et al. Signaling through OX40 enhances antitumor immunity. Semin Oncol 2010;37:524-32.
29. Zaini J, Andarini S, Tahara M, et al. OX40 ligand expressed by DCs costimulates NKT and CD4+ Th cell antitumor immunity in mice. J Clin Invest 2007;117:3330-8.

30. Farkona S, Diamandis EP, Blasutig IM. Cancer immunotherapy: the beginning of the end of cancer? $\mathrm{BMC}$ Med 2016;14:73.

31. Liu J, Hamrouni A, Wolowiec D, et al. Plasma cells from multiple myeloma patients express B7-H1 (PD-L1) and increase expression after stimulation with IFN-\{gamma $\}$ and TLR ligands via a MyD88, TRAF6-, and MEK-dependent pathway. Blood 2007;110:296-304.

32. Metelitsa LS. Anti-tumor potential of type-I NKT cells against CD1d-positive and CD1d-negative tumors in humans. Clin Immunol 2011;140:119-29.

33. Croft M. Control of immunity by the TNFRrelated molecule OX40 (CD134). Annu Rev Immunol 2010;28:57-78.

34. Weinberg AD, Morris NP, Kovacsovics-Bankowski M, et al. Science gone translational: the OX40 agonist story. Immunol Rev 2011;244:218-31.

35. Redmond WL, Weinberg AD. Targeting OX40 and OX40L for the treatment of autoimmunity and cancer. Crit Rev Immunol 2007;27:415-36.

36. Zhang N, Sadun RE, Arias RS, et al. Targeted and untargeted CD137L fusion proteins for the immunotherapy of experimental solid tumors. Clin Cancer Res 2007;13:2758-67.

37. Melero I, Shuford WW, Newby SA, et al. Monoclonal antibodies against the 4-1BB T-cell activation molecule eradicate established tumors. Nat Med 1997;3:682-5.

38. Melero I, Bach N, Hellström KE, et al. Amplification of tumor immunity by gene transfer of the co-stimulatory 4-1BB ligand: synergy with the CD28 co-stimulatory pathway. Eur J Immunol 1998;28:1116-21.

39. Pratico ED, Sullenger BA, Nair SK. Identification and characterization of an agonistic aptamer against the $\mathrm{T}$ cell costimulatory receptor, OX40. Nucleic Acid Ther 2013;23:35-43.

40. Dollins CM, Nair S, Boczkowski D, et al. Assembling OX40 aptamers on a molecular scaffold to create a receptor-activating aptamer. Chem Biol 2008;15:675-82.

41. Nozari A, Berezovski MV. Aptamers for CD Antigens: From Cell Profiling to Activity Modulation. Mol Ther Nucleic Acids 2017;6:29-44.

42. Song Y, Margolles-Clark E, Bayer A, et al. Smallmolecule modulators of the OX40-OX40 ligand co- 
stimulatory protein-protein interaction. Br J Pharmacol 2014;171:4955-69.

43. Salem ML, El-Naggar SA, Cole DJ. Cyclophosphamide induces bone marrow to yield higher numbers of precursor dendritic cells in vitro capable of functional antigen presentation to $\mathrm{T}$ cells in vivo. Cell Immunol 2010;261:134-43.

44. North RJ. Cyclophosphamide-facilitated adoptive immunotherapy of an established tumor depends on elimination of tumor-induced suppressor T cells. J Exp Med 1982;155:1063-74.

45. Doloff JC, Chen CS, Waxman DJ. Anti-tumor innate immunity activated by intermittent metronomic cyclophosphamide treatment of 9L brain tumor xenografts is preserved by anti-angiogenic drugs that spare VEGF receptor 2. Mol Cancer 2014;13:158.

46. Ailles LE, Weissman IL. Cancer stem cells in solid tumors. Curr Opin Biotechnol 2007;18:460-6.

47. Wright MH, Calcagno AM, Salcido CD, et al. Brca1 breast tumors contain distinct CD44+/CD24- and CD133+ cells with cancer stem cell characteristics. Breast Cancer Res 2008;10:R10.

48. Hermann PC, Huber SL, Herrler T, et al. Distinct populations of cancer stem cells determine tumor growth and metastatic activity in human pancreatic cancer. Cell Stem Cell 2007;1:313-23.

49. Dylla SJ, Beviglia L, Park IK, et al. Colorectal cancer stem cells are enriched in xenogeneic tumors following chemotherapy. PLoS One 2008;3:e2428.

50. Bao S, Wu Q, McLendon RE, et al. Glioma stem cells promote radioresistance by preferential activation of the DNA damage response. Nature 2006;444:756-60.

51. Phillips TM, McBride WH, Pajonk F. The response of CD24(-/low)/CD44+ breast cancer-initiating cells to radiation. J Natl Cancer Inst 2006;98:1777-85.

52. Yu F, Yao H, Zhu P, et al. let-7 regulates self renewal and tumorigenicity of breast cancer cells. Cell 2007;131:1109-23.

53. Wicha MS. Cancer stem cell heterogeneity in hereditary breast cancer. Breast Cancer Res 2008;10:105.

54. Ibe S, Qin Z, Schüler T, et al. Tumor rejection by disturbing tumor stroma cell interactions. J Exp Med 2001;194:1549-59.

55. Bonmort M, Dalod M, Mignot G, et al. Killer dendritic cells: IKDC and the others. Curr Opin Immunol 2008;20:558-65.

56. Salem ML, Díaz-Montero CM, Al-Khami AA, et al. Recovery from cyclophosphamide-induced lymphopenia results in expansion of immature dendritic cells which can mediate enhanced prime-boost vaccination antitumor responses in vivo when stimulated with the TLR3 agonist poly(I:C). J Immunol 2009;182:2030-40.

57. Salem ML, Kadima AN, El-Naggar SA, et al. Defining the ability of cyclophosphamide preconditioning to enhance the antigen-specific CD8+ T-cell response to peptide vaccination: creation of a beneficial host microenvironment involving type I IFNs and myeloid cells. J Immunother 2007;30:40-53.

58. Salem ML, Al-Khami AA, El-Naggar SA, et al. Cyclophosphamide induces dynamic alterations in the host microenvironments resulting in a Flt3 liganddependent expansion of dendritic cells. J Immunol 2010;184:1737-47.

59. Sefc L, Psenák O, Sýkora V, et al. Response of hematopoiesis to cyclophosphamide follows highly specific patterns in bone marrow and spleen. J Hematother Stem Cell Res 2003;12:47-61.

60. Radojcic V, Bezak KB, Skarica M, et al. Cyclophosphamide resets dendritic cell homeostasis and enhances antitumor immunity through effects that extend beyond regulatory T cell elimination. Cancer Immunol Immunother 2010;59:137-48.

61. Moskalenko M, Pan M, Fu Y, et al. Requirement for innate immunity and $\mathrm{CD} 90^{+} \mathrm{NK} 1.1^{-}$lymphocytes to treat established melanoma with chemo-immunotherapy. Cancer Immunol Res 2015;3:296-304.

62. Berd D, Mastrangelo MJ. Effect of low dose cyclophosphamide on the immune system of cancer patients: depletion of CD4+, 2H4+ suppressor-inducer T-cells. Cancer Res 1988;48:1671-5.

63. Heylmann D, Bauer M, Becker H, et al. Human CD4+CD25+ regulatory $T$ cells are sensitive to low dose cyclophosphamide: implications for the immune response. PLoS One 2013;8:e83384.

64. Petty AJ, Yang Y. Tumor-associated macrophages: implications in cancer immunotherapy. Immunotherapy 2017;9:289-302.

65. Corzo CA, Condamine T, Lu L, et al. HIF-1 $\alpha$ regulates function and differentiation of myeloid-derived suppressor cells in the tumor microenvironment. J Exp Med 2010;207:2439-53.

66. Kumar V, Cheng P, Condamine T, et al. CD45 Phosphatase Inhibits STAT3 Transcription Factor Activity in Myeloid Cells and Promotes TumorAssociated Macrophage Differentiation. Immunity 2016;44:303-15. 
67. Li H, Han Y, Guo Q, et al. Cancer-expanded myeloidderived suppressor cells induce anergy of NK cells through membrane-bound TGF-beta 1. J Immunol 2009;182:240-9.

68. Gabrilovich DI. Myeloid-Derived Suppressor Cells. Cancer Immunol Res 2017;5:3-8.

69. Medina-Echeverz J, Aranda F, Berraondo P. Myeloidderived cells are key targets of tumor immunotherapy. Oncoimmunology 2014;3:e28398.

70. Doloff JC, Waxman DJ. VEGF receptor inhibitors block the ability of metronomically dosed cyclophosphamide to activate innate immunity-induced tumor regression. Cancer Res 2012;72:1103-15.

71. Palma JP, Aggarwal SK. Cisplatin and carboplatin mediated release of cytolytic factors in murine peritoneal macrophages in vitro. Anticancer Drugs 1994;5:615-22.

72. Medina-Echeverz J, Fioravanti J, Zabala M, et al. Successful colon cancer eradication after chemoimmunotherapy is associated with profound phenotypic change of intratumoral myeloid cells. J Immunol 2011;186:807-15.

73. Berraondo P, Nouzé C, Préville X, et al. Eradication of large tumors in mice by a tritherapy targeting the innate, adaptive, and regulatory components of the immune system. Cancer Res 2007;67:8847-55.

74. Guiducci C, Vicari AP, Sangaletti S, et al. Redirecting in vivo elicited tumor infiltrating macrophages and dendritic cells towards tumor rejection. Cancer Res 2005;65:3437-46.

75. Tsung K, Dolan JP, Tsung YL, et al. Macrophages as effector cells in interleukin 12-induced T cell-dependent tumor rejection. Cancer Res 2002;62:5069-75.

76. Kisaretova PE, Kirikovich SS, Ritter GS, et al. Approbation of the Cancer Treatment Approach Based on the Eradication of TAMRA+ Cancer Stem Cells in a Model of Murine Cyclophosphamide Resistant Lymphosarcoma. Anticancer Res 2020;40:795-805.

77. Dolgova EV, Proskurina AS, Potter EA, et al. Evaluation of a strategy for tumor-initiating stem cell eradication in primary human glioblastoma cultures as a model. Vavilovskii Zhurnal Genet Selektsii 2018;22:825-36.

78. Dolgova EV, Potter EA, Proskurina AS, et al. Evaluating the effectiveness of the tumor-initiating stem cells eradication strategy on the example of human glioblastoma cell line U87. Voprosy Onkologii 2019;65:904-19.

79. Potter EA, Ritter GS, Dolgova EV, et al. Evaluating the efficiency of the tumor-initiating stem cells eradication strategy on the example of ascite form of mouse gepatocarcinoma G-29. Voprosy onkologii 2018;64:818-29.

80. Dolgova EV, Shevela EY, Tyrinova TV, et al. Nonadherent Spheres With Multiple Myeloma Surface Markers Contain Cells that Contribute to Sphere Formation and Are Capable of Internalizing Extracellular Double-Stranded DNA. Clin Lymphoma Myeloma Leuk 2016;16:563-76.

81. Dolgova EV, Petrova DD, Proskurina AS, et al. Identification of the xenograft and its ascendant sphereforming cell line as belonging to EBV-induced lymphoma, and characterization of the status of sphere-forming cells. Cancer Cell Int 2019;19:120.

82. The Russian Foundation for Basic Research project No 18-29-09045. 2018-2021.

83. Vozny EK, Dobrovolskaya NY, Gurov SN, et al. Neoadjuvant treatment of breast cancer. III Annual Russian Oncological Conference. St. Petersburg: 1999. P66-72.

84. Alyamkina EA, Nikolin VP, Popova NA, et al. Combination of cyclophosphamide and double-stranded DNA demonstrates synergistic toxicity against established xenografts. Cancer Cell Int 2015;15:32.

85. Orishchenko KE, Ryzhikova SL, Druzhinina YG, et al. Effect of human double-stranded DNA preparation on the production of cytokines by dendritic cells and peripheral blood cells from relatively healthy donors. Cancer Ther 2013;8:191-205.

86. Proskurina AS, Spaselnikova AV, Ritter GS, et al. Features of monocyte-derived dendritic cells encompassing a rare subpopulation of cells that are capable of natural internalization of extracellular dsDNA. Eur Cytokine Netw 2019;30:43-58.

87. Proskurina AS, Ruzanova VS, Tyrinova TV, et al. The Basis of Anticancer Immunity Mechanism Induced by In Situ Vaccination. J Sib Fed Univ Biol 2020;2020:235-69.

88. Dolgova EV, Proskurina AS, Nikolin VP, et al. "Delayed death" phenomenon: a synergistic action of cyclophosphamide and exogenous DNA. Gene 2012;495:134-45.

89. Wang W, Erbe AK, Hank JA, et al. NK Cell-Mediated Antibody-Dependent Cellular Cytotoxicity in Cancer Immunotherapy. Front Immunol 2015;6:368.

90. Geffner JR. Antibody dependent cellular cytotoxicity. In: Encyclopedia of Immunology. Academic Press; 1998:168-71.

91. Gómez Román VR, Murray JC, Weiner LM. AntibodyDependent Cellular Cytotoxicity (ADCC). Antibody Fc 
2014;1-27.

92. Igietseme JU, Zhu X, Black CM. Fc Receptor-Dependent Immunity. Antibody Fc 2014:269-81.

Cite this article as: Proskurina AS, Ruzanova VS, Ostanin AA, Chernykh ER, Bogachev SS. Theoretical premises of a "three in one" therapeutic approach to treat immunogenic and nonimmunogenic cancers: a narrative review. Transl Cancer Res 2021;10(11):4958-4972. doi: 10.21037/tcr-21-919
93. Croft M, So T, Duan W, et al. The significance of OX40 and OX40L to T-cell biology and immune disease. Immunol Rev 2009;229:173-91. 